\title{
Model-based sound synthesis of the guqin
}

\author{
Henri Penttinen, a) Jyri Pakarinen, and Vesa Välimäki \\ Laboratory of Acoustics and Audio Signal Processing, Helsinki University of Technology, Espoo, Finland \\ Mikael Laurson \\ Centre for Music and Technology, Sibelius Academy, Helsinki, Finland
}

\author{
Henbing Li and Marc Leman \\ Institute for Psychoacoustics and Electronic Music, Department of Musicology, Ghent University, Ghent, \\ Belgium
}

(Received 5 May 2006; revised 14 September 2006; accepted 14 September 2006)

\begin{abstract}
This paper presents a model-based sound synthesis algorithm for the Chinese plucked string instrument called the guqin. The instrument is fretless, which enables smooth pitch glides from one note to another. A version of the digital waveguide synthesis approach is used, where the string length is time-varying and its energy is scaled properly. A body model filter is placed in cascade with the string model. Flageolet tones are synthesized with the so-called ripple filter structure, which is an FIR comb filter in the delay line of a digital waveguide model. In addition, signal analysis of recorded guqin tones is presented. Friction noise produced by gliding the finger across the soundboard has a harmonic structure and is proportional to the gliding speed. For pressed tones, one end of a vibrating string is terminated either by the nail of the thumb or a fingertip. The tones terminated with a fingertip decay faster than those terminated with a thumb. Guqin tones are slightly inharmonic and they exhibit phantom partials. The synthesis model takes into account these characteristic features of the instrument and is able to reproduce them. The synthesis model will be used for rule based synthesis of guqin music. (C) 2006 Acoustical Society of America.
\end{abstract}

[DOI: 10.1121/1.2360422]

PACS number(s): 43.75.Gh, 43.75.Wx [NHF]

Pages: 4052-4063

\section{INTRODUCTION}

The purpose of this paper is to provide an insight to the acoustical characteristics of the guqin and to propose a model-based synthesis algorithm that is simple enough to run in real time.

The guqin (pronounced ku-ch' in), also called the sevenstrings-qin, is the modern name for the fretless plucked string instrument qin, is the oldest Chinese string instrument still used in modern times. ${ }^{1,2}$ The current structure of the instrument was formed approximately between the 5th and the 7 th centuries, and since then there have been no major changes in the construction. Figure 1 shows the guqin in an in situ fashion in the small anechoic chamber at Helsinki University of Technology. This paper proposes a physically inspired sound synthesis technique to generate guqin tones and music using a computer.

The guqin is one of the most important topics in Chinese musicological studies, and due to its long history, rich documentation, and precious old musical notation, there is a good understanding of the role of the guqin in Chinese culture. ${ }^{3}$ In contrast, the acoustics of the instrument, as well as the guqin playing techniques, are far less well understood. In addition, transforming of the large body of traditional guqin tabulature into music, i.e., modern music notation and sound, has been a difficult task. The proposed guqin sound synthesizer is the first step in the process of digitizing existing guqin music.

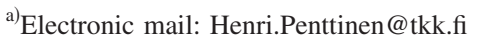

With a suitable piece of software, a computer system can be an efficient tool for the guqin tabulature transcription making the work much easier and faster than manual labor. Additionally, a virtual musical instrument system enables composers to create new music for the guqin and extends the possibilities of composing music with new playing styles.

The proposed guqin synthesizer is based on the commuted digital waveguide synthesis technique. ${ }^{4,5}$ The digital waveguide technique has been used successfully to synthesize a broad range of traditional and ethnic instruments. ${ }^{6-8}$ Since the length of the string is varied in time the instrument model cannot be a purely commuted one. Hence, a body model filter is placed in cascade with the string model. This way the modulation of the body response with the timevarying fundamental frequency is partly avoided. Previously, a neural network based synthesis model for the guqin has been proposed. ${ }^{9}$

The model-based synthesis proposed here takes into account the important characteristics of guqin playing and acoustics, namely, flageolet tones, also called harmonics, gliding of tones, two different ways of terminating the string, and phantom partials. ${ }^{10}$ A systematic discussion on the synthesis of flageolet tones has previously been reported. ${ }^{11}$ Here, the ripple filter ${ }^{12}$ is used for flageolet tone synthesis and a systematic calibration method is introduced. During gliding of tones, the friction noise produced has a harmonic structure and is proportional to the gliding speed. Previously, several computational models for friction sound simulation have been presented. ${ }^{13-15}$ See, e.g., Ref. 16 for an overview. For 


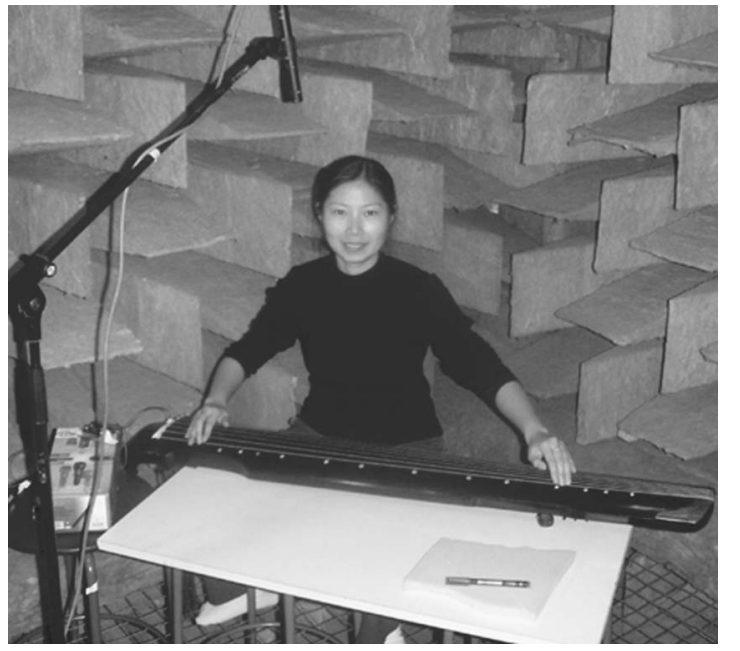

FIG. 1. Ms. Li playing the guqin in the small anechoic chamber at Helsinki University of Technology.

the purposes here a kinetic friction model in the vein of Cook's model-based synthesizers ${ }^{17}$ is introduced. Also, due to gliding of tones the string model has to be energetically compensated as described by Pakarinen et al. ${ }^{18}$ The finger surface, the nail or the fingertip, that terminates the string affects the behavior of the tone and is accounted for in the synthesis model.

Additionally, based on the analysis of the guqin tones it has become evident that the string vibration exhibits phantom partials. ${ }^{10}$ The first systematic report on the splitting behavior appeared for the piano by Nakamura and Naganuma ${ }^{19}$ and later for the guitar by Woodhouse. ${ }^{20}$ Bank and Sujbert explain this to be a result of transverse to longitudinal coupling which is of a nonlinear nature. ${ }^{21}$ Many synthesis models for this phenomenon have been proposed recently. ${ }^{21-23}$ A computationally efficient solution, that follows the one discussed by Bank and Sujbert, ${ }^{22}$ is proposed here.

The remainder of this paper is organized as follows: The structure, tuning, and playing techniques of the instrument are discussed in Sec. II. Section III presents the results of signal analysis of recorded guqin tones to illustrate the characteristics of the timbre of the instrument. The analyzed features include the initial pitch glide of tones, the inharmonicity caused by string stiffness, effects of nail and fingertip terminations of the string, and the friction sounds produced by sliding the finger along the string during gliding tones. In Sec. IV, the waveguide synthesis algorithm, which is loosely based on the physics of the instrument, is introduced. Particular care is paid to the faithful generation of clean gliding tones and realistic friction noise, whose characteristics vary according to the speed of pitch change. Also, the playing of harmonics, which is commonly used in guqin music, can be imitated using the algorithm. Synthetic tones are compared against recordings with the help of signal analysis. Section V addresses the implementation of the synthesizer using a software system developed for physics-based sound synthesis. The software includes a music notation tool called ENP (Expressive Notation Package) that enables the writing and playing of large pieces of computer-generated guqin music.

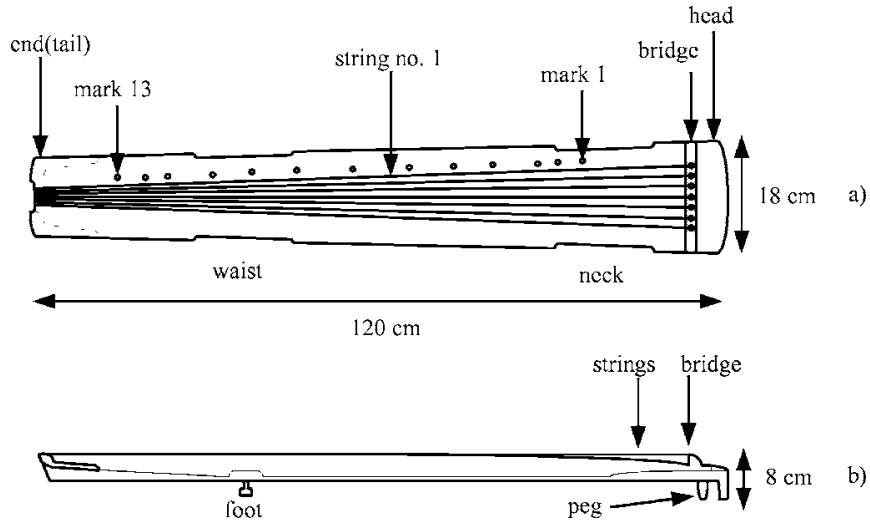

FIG. 2. Construction of the guqin from two angles: (a) top view and (b) side view.

Within ENP, the musical performance parameters, such as timing, dynamics, and playing styles, can be adjusted either manually or by assigning automatic rules.

\section{DESCRIPTION OF THE INSTRUMENT}

Next, the construction and playing style of the guqin is briefly described.

\section{A. Construction and tuning}

The body of the guqin is a long, narrow, hollow box made from two pieces of wooden board, and the top board is carved into an arch while the bottom is flat (see Figs. 1 and 2 ). For the top board soft wood is usually used (such as tung), while the wood for the bottom board is hard (catalpa or fir). There are two sound holes in the bottom board, and, directly above, on the inner side of the top board there are two protrusions with the same shape as the sound holes.

The surface of the box is covered with a special layer (about $1 \mathrm{~mm}$ ) of roughcast, which is a mixture of deer horn powder (or bone powder or tile powder) and raw lacquer, and there are several layers of raw lacquer along the top of the roughcast for polishing.

The bridge is made from hard wood, and the strings are attached to it with a twisting-rope system, which allows finetuning of the strings in a limited range. The other ends of the strings are bent over the end (tail) and are finally tied up to the feet on the bottom. Traditionally, the strings were made of silk, but after the 1950s they have been replaced mainly by steel-nylon strings. There are 13 marks inlayed on the roughcast at the side of the first string, which indicate the positions of the first to the fifth and the seventh overtone. These marks also function as a reference for stopped strings, i.e., when the string is pressed against the top board. Each part between two contiguous marks is divided into ten parts, for example, mark 2.5 indicates that the tone is played halfway between the second and the third mark.

The seven strings are tuned basically as a pentatonic scale. The basic tuning of the open strings is $\mathrm{C}_{2}, \mathrm{D}_{2}, \mathrm{~F}_{2}, \mathrm{G}_{2}$, $A_{2}, C_{3}$, and $D_{3}$ from the lowest string (No. 1) to the highest (No. 7). The pitch range for so called stopped strings is from $65.2 \mathrm{~Hz}$ (open string No. 1) to $787.5 \mathrm{~Hz}$ (string No. 7, mark 2.6) which roughly correspond to notes $\mathrm{C}_{2}$ and $\mathrm{G}_{5}$, respec- 
tively. The highest harmonic or flageolet sound is played on string No. 7 on marks No. 1 or No. $13\left(f_{0}=1174.7 \mathrm{~Hz}, \mathrm{D}_{6}\right)$.

The guqin used in this measurement was made by Zhang Jianhua in Beijing in 1999. The boards are made of fir, and the roughcast is deer horn powder and raw lacquer. Shangyin steel-nylon strings are used with the following diameters in $\mathrm{mm}$ from string No. 1 to string No. 7: (1) 1.38, (2), 1.20, (3) 1.08 , (4) 1.00 , (5) 0.90 , (6) 0.75 , (7) 0.64 . The mass density for strings Nos. 5 and 7 were measured to be $1.32 \mathrm{~g} / \mathrm{m}$ and $0.66 \mathrm{~g} / \mathrm{m}$, respectively.

\section{B. Playing techniques}

In modern days the guqin is usually played on a table with its two feet standing on the table and the neck laying on the right edge of the table with antislip mats between the contact points of the table and the instrument (see Fig. 1). The right hand plucks the strings between the bridge and the first mark, and the left hand presses the strings against the top plate of the body. The instrument is fretless, which enables smooth sliding tones. Guqin music also incorporates substantial use of harmonics or flageolet tones.

The little fingers of neither hand are used. The other four fingers of the right hand pluck the string from both the fleshy and the nail side. Typically, the nail exceeding the finger is 2-3 mm long for the thumb and 1-2 $\mathrm{mm}$ long for the other fingers. The left thumb presses the string on the right side, where the nail and flesh joins, or at the first joint. The other three left fingers press the string with the fleshy top part of the finger or occasionally with the left side of the first ring finger joint.

\section{ACOUSTIC MEASUREMENTS AND SIGNAL ANALYSIS}

To creat a synthesizer for the instrument, an extensive set of isolated plucks was recorded. The purpose of the isolated plucks is to be able to properly analyze the characteristics of the instrument.

\section{A. Measurement and recording setup}

Guqin tones were recorded in the small anechoic chamber of Helsinki University of Technology. The recordings were made with a microphone (AKG C 480 B, cardioid capsule) placed at a distance of about $1 \mathrm{~m}$ above the sound board (see Fig. 1). The signals were recorded digitally $(44.1 \mathrm{kHz}, 16$ bits) with a digital mixer (Yamaha 01v) and soundcard (Digigram VX Pocket) onto the hard drive of a PC laptop. To remove infrasonic disturbances the signals were highpass filtered with a fourth-order Butterworth filter with a cutoff frequency of $52 \mathrm{~Hz}$.

As for the tones, four complete sets on a typical scale used in guqin music were recorded. Two different styles for terminating the string with the left-hand finger were used, the nail of the thumb or the fingertip of other fingers. In the following text these styles of string termination will be referred to as nail or fingertip. Furthermore, two different plucking styles were recorded, namely, plucking with the middle finger towards the player or plucking with the index finger away from the player. In addition, a complete set of

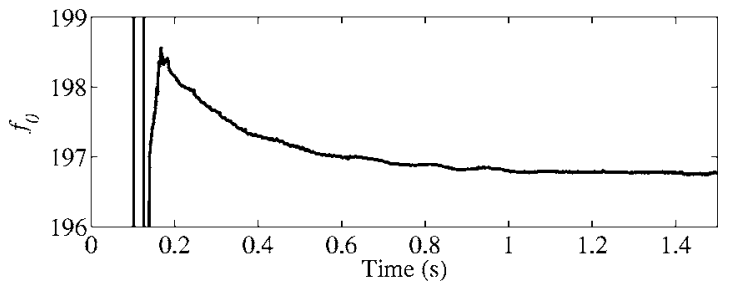

FIG. 3. Initial pitch glide of a forte fortissimo tone played on the guqin (string No. 4, mark 7).

harmonics or flageolet tones was recorded for all strings and marks. Moreover, three dynamic levels (pp, $\mathrm{mf}$, and ff) were recorded for all open strings and marks seven. Slides, isolated vibratos, scales, and musical pieces were also included in the database. Important for this study are the basic pluck events (281 samples), sliding sounds, and the harmonic sounds (91 samples) that will be analyzed next. All in all, the database contains over 400 samples.

\section{B. Analysis of guqin tones}

Next, the essential features in the behavior of guqin tones are illustrated. Their prominent patterns in the time and frequency domain, i.e., the initial pitch glide, decay of sound with different termination, and inharmonicity, are discussed. The discussion of flageolet tones is presented together with its synthesis and results in Secs. IV B and IV C.

\section{Initial pitch glide}

Initial pitch glide is a phenomenon due to tension modulation and occurs in vibrating strings. ${ }^{24-26}$ Even a small transverse displacement of the string causes a second-order change in its length, and therefore in its tension. This causes the pitch to decay after releasing the string from its initial displacement. Hence, some initial pitch gliding occurs in guqin tones. The amount of pitch gliding for tones played as mezzoforte and forte fortissimo notes were measured.

For mezzoforte tones the largest initial pitch glide value obtained was 0.075 ERB (Equivalent Rectangular Bandwidth), ${ }^{27}$ while the mean was 0.025 ERB with a standard deviation of 0.021. The number of ERBs is defined as $21.4 \log _{10}(4.37 F+1)$, where $F$ is frequency in $\mathrm{kHz}^{27}$ Similarly, for forte fortissimo tones the mean value for the initial pitch glides was 0.034 ERB with a standard deviation of 0.019. The largest value was 0.096 ERB (for string 5, mark 7). According to Järveläinen ${ }^{28}$ these initial pitch glides would remain inaudible to most listeners, since the limit for the initial pitch glide audibility is about 0.1 ERB. However, the quartile limits are quite large and hence expert listeners, such as instrument players, are able to detect smaller changes than $0.1 \mathrm{ERB}^{28}$

Figure 3 shows the behavior of the fundamental frequency $f_{0}$ in time for a forte fortissimo tone played on string No. 4, mark 7 (about $\mathrm{G}_{3}$ ). The $x$ axis displays time and the $y$ axis displays the fundamental frequency. At $0.18 \mathrm{~s}$ the fundamental frequency is $198.6 \mathrm{~Hz}$ and beyond $1 \mathrm{~s}$ it is $197 \mathrm{~Hz}$. This gives a change of $1.6 \mathrm{~Hz}$, which is 0.035 ERB. The fundamental frequency estimations have been calculated 


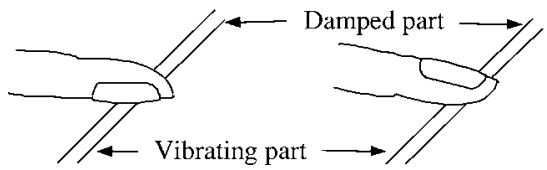

FIG. 4. Sketch of string termination with (a) the nail of the thumb and (b) the fingertip part of the forefinger.

with the autocorrelation-based YIN algorithm. ${ }^{29}$ The glitch in Fig. 3 is due to estimation errors during the attack, i.e., when $t<0.18 \mathrm{~s}$.

The largest measured initial pitch glide can be audible for some listeners, therefore the initial pitch glides are synthesized with the obtained mean value, i.e., 0.025 ERB for $m f$ tones and 0.034 ERB for $f f$ tones. The initial pitch glides are synthesized by changing the pitch of the string with a break point function in the PWGLSynth system. ${ }^{30}$ Alternatively, the synthesis could use the nonlinear approach proposed by Tolonen et al. ${ }^{26}$

\section{Behavior of tones for nail and fingertip terminations}

Two interesting and fundamental phenomena regarding the behavior of the harmonics and their decay were found. First, in guqin playing either the nail of the thumb or the fingertip of another finger terminates stopped strings. This causes a difference in decay times. Secondly, phantom partials were found. The analysis of these phenomena are presented next.

Figure 4 shows a sketch of where the string is terminated with (a) the nail of the thumb and (b) the fingertip of the forefinger. Figure 5(a) presents the time response of a guqin tone played on string 6 mark 5 which is terminated by the nail $\left(f_{0}=392.25 \mathrm{~Hz}\right)$. The corresponding plot for a tone terminated by the fingertip is depicted in Fig. 5(b) $\left(f_{0}\right.$ $=392.44 \mathrm{~Hz}$ ). Comparing the time responses shows that the string terminated by the nail [Fig. 5(a)] has a steady decay with very slight amplitude modulation. The tone terminated with the fingertip [Fig. 5(b)] exhibits a rapid decay shortly after the attack of the tone and then a steady, slower decay.
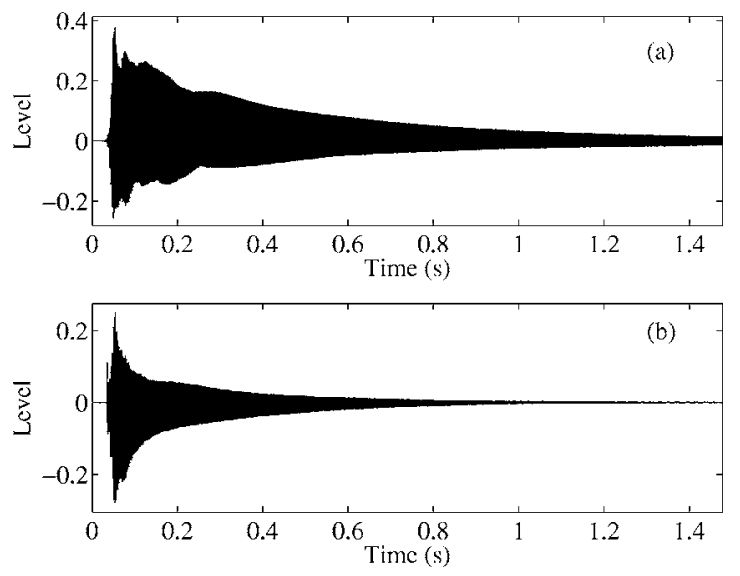

FIG. 5. Time responses of guqin tones played on string 6 mark 5, when (a) the nail of the thumb terminates the string and (b) the fingertip of the forefinger terminates the string.
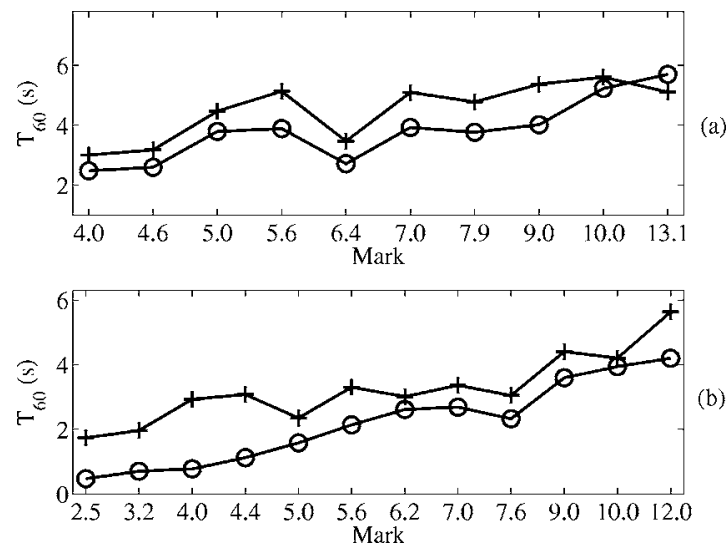

FIG. 6. $T_{60}$ times for all nail $(+)$ and fingertip $(\bigcirc)$ terminated tones for (a) string 1 and (b) string 7, where the $x$ axis indicates the mark (string length).

The decay times of the tones terminated with nail and fingertip are illustrated in Fig. 6. The figure shows the $T_{60}$ times as a function of the mark, i.e., the $y$ axis indicates the decay times and the $x$ axis where string was pressed from. The results for string 1 are shown in pane (a) and for string 7 in (b). The termination style is indicated as follows, nail with $(+)$ and fingertip with (o). Figure 6 indicates clearly that the decay times of fingertip tones are smaller than those of the nail tones. The reason behind the discovered differences is, naturally, due to a difference in the way the string is terminated with the left hand. When the nail terminates the string, the losses are smaller than when the fingertip terminates the string.

Figure 7 reveals the existence of phantom partials for tones played on string 6 mark 5. Spectral peaks have been manually picked and only spectral peaks that could be reliably identified from the spectrum are shown. Therefore, in Fig. 7(b), peaks above $5 \mathrm{kHz}$ are not shown. The splitting phenomena occur for both nail and fingertip termination. The mode splitting follows the inharmonicity factor $B$ (solid line) and $B / 4$ (dashed line) relation, first reported for the piano by

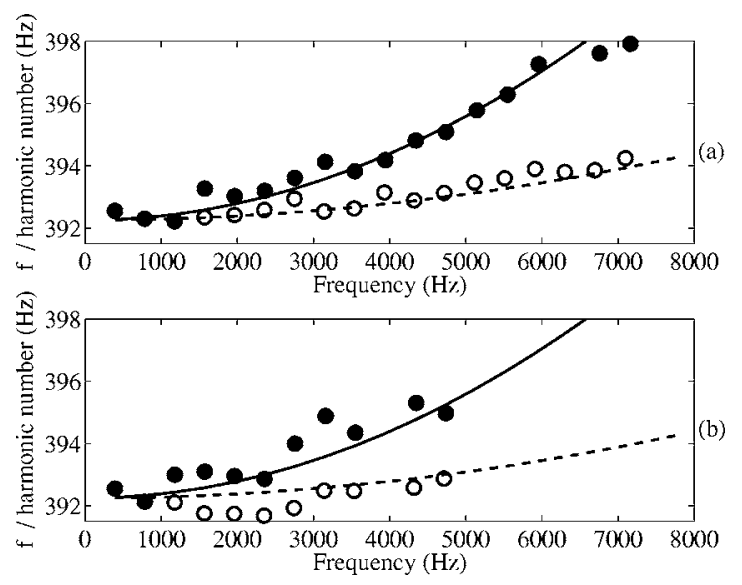

FIG. 7. Splitting of modes for string 6 mark 5, when the (a) nail of the thumb terminates the string and (b) the fingertip of the forefinger terminates the string. Black dots follow the inharmonicity value $B=0.00009$ (solid line) and the white dots follow the $B / 4$ trend (dashed line). Fundamental frequencies obtained with the YIN algorithm for tones in panels (a) and (b) are $392.25 \mathrm{~Hz}$ and $392.44 \mathrm{~Hz}$, respectively. 

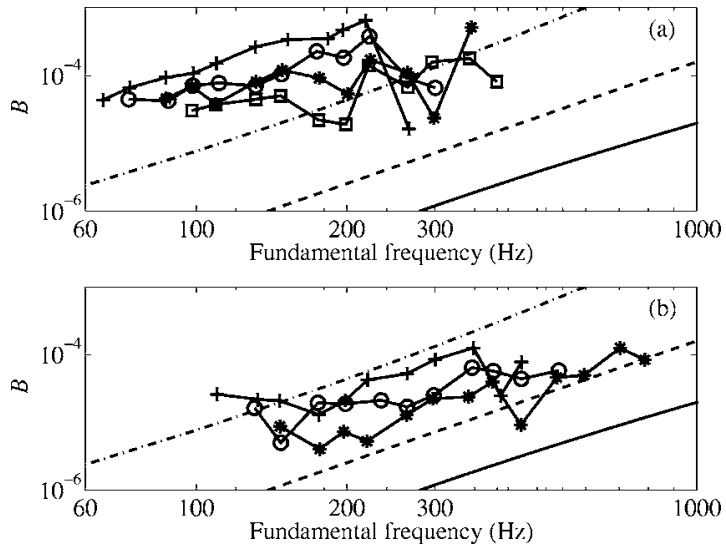

FIG. 8. Estimates of the inharmonicity coefficient $B$ analyzed from recorded guqin tones. Panel (a) shows strings 1-4 and (b) strings 5-7. All seven strings and recorded notes have been covered, hence the overlapping. Strings are presented with the following symbols: in subplot (a) No. 1 with $(+)$, No. 2 with $(\bigcirc)$, No. 3 with (*), and No. 4 with $(\square)$, and in subplot (b) No. 5 with $(+)$, No. 6 with $(\bigcirc)$, No. 7 with (*). The threshold of audibility is plotted as a dashed line with its $90 \%$ confidence intervals as dasheddotted and solid lines (Ref. 31).

Nakamura and Naganuma. ${ }^{19}$ Hence, the fingertip termination can be considered as increasing losses, but does not prevent the generation of phantom partials.

\section{Inharmonicity}

The inharmonicity of guqin tones was investigated for all strings and recorded marks. Inharmonicity for the guqin is depicted in Fig. 8 as a function of frequency, on a log-log scale. The threshold of audibility according to Järveläinen $e t$ $a l^{31}$ is also plotted as a dashed line with its $90 \%$ confidence intervals as dashed-dotted and solid lines. Results for all seven strings and stopped tones are depicted, and, therefore, overlapping of frequencies occurs.

As for the inharmonicity, two observations can be made. First, the inharmonicity for lower strings (strings 1-4) is larger than for higher strings (strings 5-7), see Figs. 8(a) and 8(b), respectively. This results from a higher Young's modulus value for steel than nylon. The lower strings are made of a combination of steel and nylon, whereas the higher strings are solely of nylon. Secondly, the inharmonicity increases as the length of the string decreases. This can be observed clearly, and especially for strings 1 and 7, as a positive slope of the data points. This is explained by the fact that the length decreases while the diameter stays fixed. This is revealed when inharmonicity is formulated $\operatorname{as}^{32} B$ $=\pi^{3} Q d^{4} /\left(64 l^{2} T\right)$, where $d$ is the diameter of the string, $l$ is its length, $Q$ is Young's modulus, and $T$ is tension. In addition, when a string is pressed against the top board or fret board, the tension of the string slightly increases due to elongation. ${ }^{33}$ This works against the increasing of inharmonicity as the string becomes shorter, but it does not compensate for the change in length versus the diameter.

According to the threshold of audibility the inharmonicity should be synthesized for at least strings $1-4$. However, a recent study shows that the threshold for audibility can be even lower for real plucked string sounds ${ }^{34}$ than previously suggested. ${ }^{31}$ Additionally, the nature of inharmonicity pre- (a)

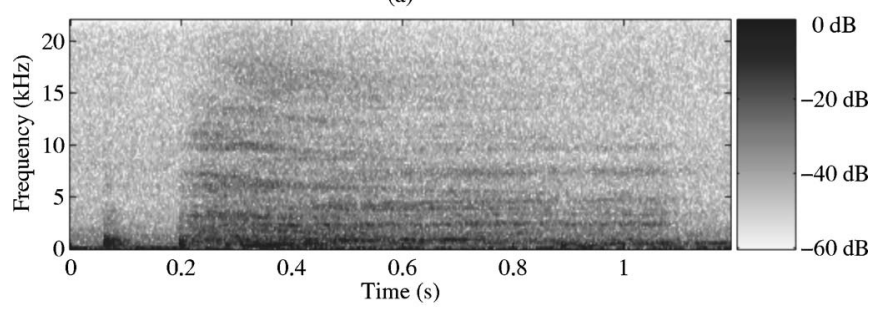

(b)

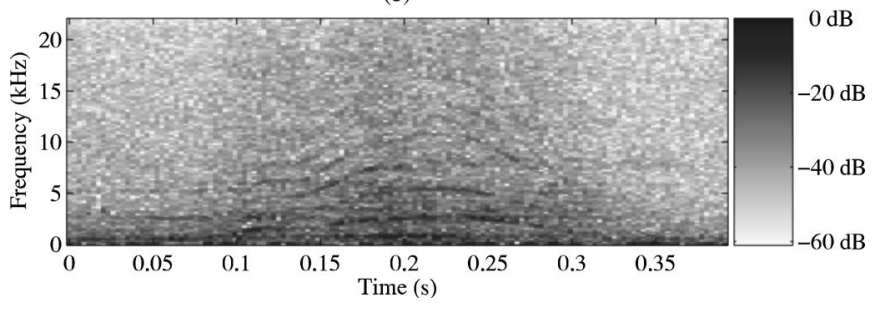

FIG. 9. (a) Spectrogram of the friction noise when sliding slowly from mark 9 to mark 7 on the 1 st string $(99-131 \mathrm{~Hz})$. (b) Spectrogram of the friction noise when sliding quickly from mark 9 to mark 7 on the 7 th string $(220-297 \mathrm{~Hz})$

vails in the time domain, as the high-frequency waves travel faster than the low-frequency waves as a result of bending stiffness. ${ }^{20}$ What is more, a beating effect has been noted to occur with low-inharmonicity conditions. ${ }^{31,35}$ In some cases, this beating effect can be a perceptual clue for detecting inharmonicity. More specifically, the beating can be a perceptual clue in relatively long tones, whereas in short tones the beating effect is not perceived, since not enough cycles of the beating occur. ${ }^{31,35}$ For these reasons, in high quality sound synthesis of the guqin tones the inharmonicity must be taken into account, while for an average listener it is questionable if it makes any difference. In waveguide models, inharmonicity is typically modeled with allpass filters. ${ }^{36-39}$ In this study the inharmonicity is synthesized with a chain of four second-order allpass filters for low tones $(349<\mathrm{Hz})$ and with a single second-order allpass filter for high tones (349 $>\mathrm{Hz})^{40}$

\section{Analysis of friction sounds}

The friction noise caused by the sliding finger-string contact was recorded using the setup described in Sec. III A. In order to record only the friction noise, the strings were not plucked. Figure 9(a) shows the spectrogram of the friction noise when the player slides her finger from mark 9 to mark 7 on the lowest string (i.e., from $99 \mathrm{~Hz}$ to $131 \mathrm{~Hz}$ ). In Fig. 9(a), the player was asked to perform the slide slowly. Figure 9(b) shows the friction noise spectrogram with a slide from mark 9 to mark 7 on the highest string (i.e., from $220 \mathrm{~Hz}$ to $297 \mathrm{~Hz}$ ). Here, the player was asked to perform the slide quickly. Note that both sliding styles still fall under the normal playing styles of the guqin, and that they represent the two sliding velocity extremes usually played on the instrument.

The spectrogram plot reveals that the friction signal is similar to lowpass-filtered noise, where the amplitude and cutoff frequency are proportional to the sliding velocity (the amplitude and cutoff frequency are highest in the middle of 


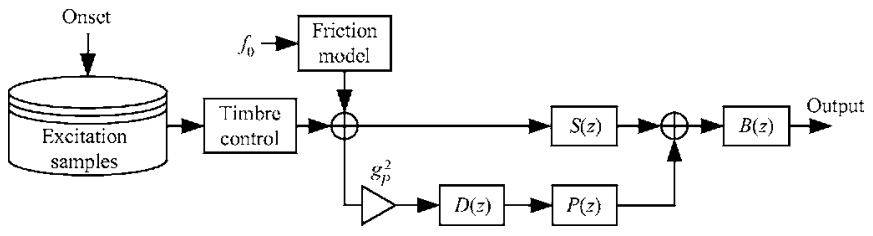

FIG. 10. Block diagram of the guqin synthesizer.

the slide, where also the sliding velocity is highest). In addition to this, there is a clearly observable harmonic structure in the noise [see Fig. 9(b)], consisting of a few modes, each approximately $200-400 \mathrm{~Hz}$ wide. The amplitudes and frequencies of these components also seem to be proportional to the slide velocity, the lowest harmonic having its frequency near $2.7 \mathrm{kHz}$ in Fig. 9(b).

The harmonic components in the friction noise imply that the friction signal is somewhat periodic. This periodicity is due to the nylon windings around the string. It is not likely that the harmonicity in the friction is caused by the free vibrations of the string, since the frequencies of the harmonics seem to be controlled by the sliding velocity rather than the string length. Based on Fig. 9(b), we can assume that the slide duration is approximately $150 \mathrm{~ms}$, and the frequency of the lowest harmonic is $2.5 \mathrm{kHz}$ on average during this slide. Since we also know that the distance between the 9th and 7 th marks is $183 \mathrm{~mm}$, we can deduce that the width of a single nylon winding should be $183 \mathrm{~mm} /(2.5 \mathrm{kHz} \times 0.15 \mathrm{~ms})$ $\approx 0.5 \mathrm{~mm}$. This is in conjunction with the observations made on the instrument.

\section{SOUND SYNTHESIS OF THE GUQIN}

\section{A. General description of synthesis model and time- varying string model}

The structure of the synthesis model is illustrated in Fig. 10. The guqin string model essentially constitutes two singledelay loop (SDL) (Ref. 41) digital waveguide (DWG) strings, $S(z)$ and $P(z)$, and a body model filter, $B(z)$. The length of the strings is varied during the synthesis run time. ${ }^{41}$ The two SDL string models, $S(z)$ and $P(z)$, synthesize the transversal vibrations and phantom partials of the tone, with the inharmonicity coefficients of $B$ and $B / 4$, respectively. The input signal is read from the excitation database. Somewhat similarly as proposed by Bank and Sujbert, ${ }^{22}$ the gain $g_{P}$ for $P(z)$ is squared to model the nonlinear dependence of the amplitude of the phantom partials. Initially, $P(z)$ is a full string model, however, as theory and measurements indicate, the phantom partials do not appear at the same frequency range or frequencies as the transverse vibrations. ${ }^{10,19-21}$ Consequently, the unwanted low and high frequencies are filtered out with a bandpass filter $D(z)$.

The string model $S(z)$ is illustrated in Fig. 11. The $z^{-L 1}$ block implements the (time varying) integer delay of the SDL. The traditional SDL blocks $H_{\mathrm{LF}}(z), F(z)$, and $A_{d}(z)$ (Ref. 41) and corresponds to the figure as follows. The $H_{\mathrm{LF}}(z)$ block is the loss filter implementing the frequency dependent decay due to losses in the string and $F(z)$ is a a third-order Lagrange filter applying the fractional part of the loop delay. $A_{d}(z)$ is the dispersion filter made of a chain of

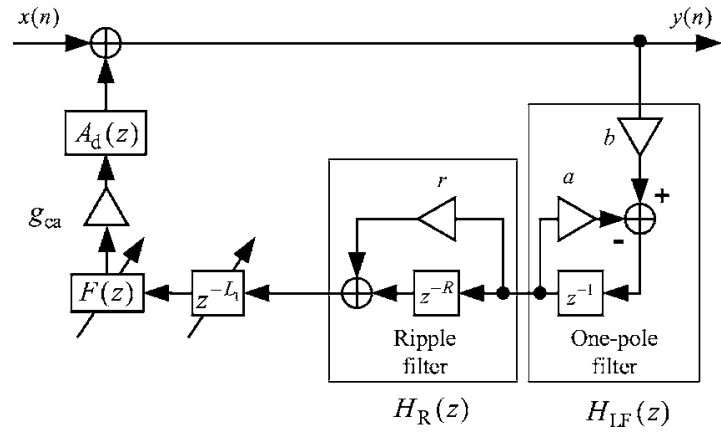

FIG. 11. Signal flow diagram of the guqin string model. The synthesis structure differs from the traditional SDL DWG model in three aspects. First, the length of the delay loop changes during run time. Secondly, the signal values are scaled by $g_{\text {ca }}$ in order to avoid the artificial changes in energy due to the pitch change. Thirdly, the ripple filter enables to synthesize flageolet tones.

four allpass filters or a single second-order allpass filter. The ripple filter ${ }^{12} H_{R}(z)$ enables efficient modelling of different decay times of partials. In the case of the guqin, the main use of the ripple filter comes in the context of flageolet tones. Coefficient $g_{\text {ca }}$ is responsible for gain compensation due to changing length of the string, as will be discussed below. When implementing the string model on a computer, the memory for the maximum length of the string should be allocated beforehand so that the possible elongation can be accounted for. The "Friction" block in Fig. 10, more thoroughly illustrated in Fig. 12, generates the friction noise emanating from the sliding finger-string contact.

\section{Energy compensation}

A problem with conventional time-varying DWGs is the fact that when the pitch of a DWG (i.e., the length of the delay loop) is changed during run time, the energy of the string is artificially altered. ${ }^{18}$ In order to fix this problem, the so-called energy compensation method ${ }^{18}$ is used, where the samples in the delay loop are scaled to compensate for the artificial energy alteration. This method was chosen over the energy preserving allpass technique discussed by Bilbao, ${ }^{42}$ since the energy compensation method is computationally less expensive and seemed to give satisfactory results for the modeling goals.

It must be noted that when simulating small pitch changes, e.g. in the case of a tension modulated string, the artificial attenuation or boosting is very likely to be negli-

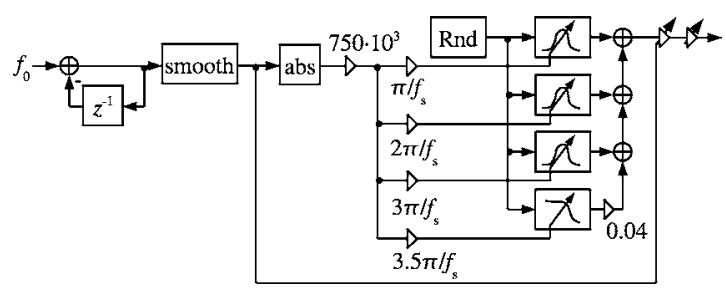

FIG. 12. Signal flow diagram of the friction sound generator. The friction sound generator consists of a noise generator (Rnd), whose output is filtered by a parallel resonator bank and lowpass-filter structure. The sliding velocity controls the central frequencies of the resonators as well as the cutoff frequency of the lowpass filter. Also, the amplitude of the friction is controlled by the slide velocity. 
gible. However, when fast and large interval slides are to be simulated, as is the case with the guqin, the artificial damping or boosting can cause audible artifacts in the synthesized sound thus needing to be compensated.

The time-varying scaling coefficient $g_{\text {ca }}$ in Fig. 11 takes care of the energy compensation when the pitch of the string is altered. It can be expressed as ${ }^{18}$

$$
g_{\text {ca }}=\sqrt{1-\Delta x},
$$

where $\Delta x$ represents the change in the delay loop length, and is evaluated as

$$
\Delta x(n)=x(n)-x(n-1),
$$

where $x$ is the delay line length and $n$ is the discrete time index. The delay line length can be easily calculated from the desired fundamental frequency $f_{0}$ of the string as

$$
x=\frac{f_{\mathrm{s}}}{2 f_{0}}-d_{\mathrm{LF}},
$$

where $f_{s}$ is the sampling frequency $\left(f_{s}=44100 \mathrm{~Hz}\right.$ was used) and $d_{\mathrm{LF}}$ is the group delay imposed by the loss filter $H_{\mathrm{LF}}(z)$ at $\mathrm{DC}$.

\section{The friction model}

As discussed in Sec. III C, the friction noise signal resembles lowpass-filtered noise, whose amplitude and cutoff frequency are proportional to the sliding velocity. More importantly, the friction sound has a few harmonic components, whose amplitudes and frequencies are also proportional to the sliding velocity. For simulating this signal, the friction model as a random noise signal, filtered by a structure of parallel resonators and a lowpass filter, was implemented. The construction of the friction block is illustrated in Fig. 12. This system can be seen as a source-filter structure, where the sound is created in a white noise generator (Rnd block), after which it is inserted into a parallel filter structure consisting of three resonators and a lowpass filter.

The resonator transfer functions are given as

$$
R_{m}(z)=\frac{(1-G)\left(1-z^{-2}\right)}{1-2 G \cos \left(\omega_{m}\right) z^{-1}+(2 G-1) z^{-2}}, \quad m=1,2,3,
$$

where

$$
G=\frac{1}{1+d}, \quad \text { and } d=\tan \left(\frac{B_{w}}{2}\right) .
$$

In the equations above, $B_{w}$ stands for the resonator bandwidth (the distance between the $-3 \mathrm{~dB}$ locations), normalized between $[0,2 \pi]$, while $\omega_{m}$ is the central frequency of resonator $n$, also normalized between $[0,2 \pi]$. The transfer function of the lowpass filter is given as

$$
H_{\mathrm{LP}}(z)=\left(\frac{1-c}{2}\right) \frac{1+z^{-1}}{1-c z^{-1}}, \quad \text { where } c=\frac{1-\sin \left(\omega_{4}\right)}{\cos \left(\omega_{4}\right)} .
$$

Here, $\omega_{4}$ is the cutoff frequency (the $-3 \mathrm{~dB}$ point) of the filter, normalized to between $[0,2 \pi]$.
The purpose of each resonator is to create a distinguishable noise component in the friction signal. When the resonators' central frequencies are set to harmonic intervals, the resulting noise signal has a harmonic structure. With this in mind, the $\omega$ terms can be written as

$$
\omega_{m}=\alpha\left|\Delta f_{0}\right| \frac{n \pi}{f_{s}}, \quad m=1,2,3
$$

for the resonators and

$$
\omega_{4}=\alpha\left|\Delta f_{0}\right| \frac{3.5 \pi}{f_{\mathrm{s}}},
$$

for the lowpass filter. In the equations above,

$$
\Delta f_{0}(n)=f_{0}(n)-f_{0}(n-1),
$$

where $f_{0}(n)$ is the fundamental frequency of the waveguide at time instant $n$, and $\alpha$ is a scaling coefficient that defines how much the pitch change signal $\Delta f_{0}(n)$ will alter the filter frequencies. It must be noted that in practical applications $\Delta f_{0}(n)$ will be quite small, since the pitch change rate is usually almost negligible when compared to the sampling rate $f_{s}$. In experiments performed, it was noted that the value $\alpha=750 \times 10^{3}$ worked well.

While testing the system, it was found that since the $\Delta f_{0}$ signal controls the filter frequencies, it should be relatively smooth and not have abrupt changes. If $\Delta f_{0}$ has a significant high-frequency content, the filter structure will produce clearly audible clicks, since the filter characteristics are changed rapidly. For this reason, the $\Delta f_{0}$ single is smoothed before controlling the filters with it. The transfer function of the smoothing operation can be given as

$$
T(z)=\left(\frac{1}{M}\right) \frac{1-z^{-(M+1)}}{1-z^{-1}},
$$

where $M$ specifies the order of the smoothing operation. In the experiments, the value $M=1000$ was used.

Finally, as can be seen in Fig. 12, the output of the friction block is scaled by the smoothed $\Delta f_{0}$ signal. This implements the friction amplitude vs sliding velocity dependence phenomenon discussed in Sec. III C. The last scaling coefficient in the signal chain is used for adjusting the overall gain of the friction noise. The user can set its value to between 0 and 100 .

\section{Body model}

The guqin synthesizer cannot purely be a commuted DWG model, since the length of the string is varied as a function of time. Therefore, a body model filter is placed in cascade with the string model. In addition, the excitation signals are filtered with the inverse of the body model filter. This way the effect of the body is roughly simulated in the time varying string.

The average of the excitation signal spectra of open strings was used to create the target body model filter. This target response was then modeled with a cascade of filters. First, the spectral envelope was modeled with a fourth-order linear prediction (LP) model. ${ }^{43}$ In this case the low-order LP model is unable to follow the spectral envelope at low fre- 


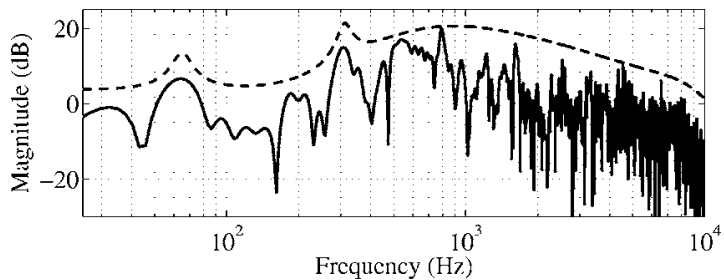

FIG. 13. Magnitude response of the body model filter (dashed line) and the target magnitude response (solid line) with a $6 \mathrm{~dB}$ offset.

quencies. Hence, a second-order shelving filter was used to attenuate the low frequencies. As a last step, two prominent low-frequency body modes are modeled with parametric second-order peak filters. The used shelving and peak filters are discussed by Zölzer ${ }^{44}$ on pp. 117-125.

Figure 13 shows the magnitude responses of the body model filter and the target response. The excitation signals fed to the instrument model are processed in advance (offline) with the inverse of the body model filter. This whitens the spectra of the excitations in the same sense as the all-pole model used in linear prediction coding whitens the excitation for the speech coding model. ${ }^{43}$ A perfect reconstruction of the effect of the body model filter is achieved with this offline processing, since when running the instrument model the body filter is in cascade with the string model. The cutoff frequency for the shelving filter is $200 \mathrm{~Hz}$ with a $20 \mathrm{~dB}$ attenuation. The peak filter parameters are $f_{c}=65 \mathrm{~Hz}$ and $310 \mathrm{~Hz}$, and $Q_{\infty}=6$ and 8, respectively, with a $10 \mathrm{~dB}$ amplification for both filters.

This body model filter approximates the spectral envelope and two low-frequency modes of the body. It is a simple and computationally efficient model. A more detailed model would include more body modes, both at low and high frequencies. This kind of resonant structure, especially at high frequencies, could be approximated with a reverb algorithm. ${ }^{12,45}$

\section{B. String model calibration}

To produce normal plucked tones the string model parameters must be calibrated as described below. First, the inharmonicity is determined, and then the excitation signals are obtained by canceling the partials of the guqin tone with a sinusoidal model. ${ }^{46}$ On the first round, the transversal vibrations are canceled and the parameters for the loss filter $H_{\mathrm{LF}}(z)$ and ripple filter $H_{R}(z)$ are obtained as described in Refs. 45 and 12, respectively. On the second round, the remaining phantom partials (longitudinal vibrations) similarly are filtered out.

In $H_{\mathrm{LF}}(z)$ the parameter $g$ controls the overall decay, and $a$ controls the frequency dependent decay. ${ }^{47}$ The transfer function is $H_{\mathrm{LF}}(z)=b /\left(1+a z^{-1}\right)$, where $b=g(1+a)$. Due to calibration errors and large differences in parameter values, for consecutive tones, the $g$ and $a$ data are smoothed in the same vein as previously proposed. ${ }^{46}$ More specifically, the $g$ parameters were treated with a 10th order median filter, and the $a$ parameters were approximated by a linear regression on the logarithmic fundamental frequency scale for each string. Additionally, the excitation signals are normalized.
This way a synthesis model was obtained that has natural and subtle changes from a tone to another without drastic unwanted sonic departures. The coefficients for the dispersion filter $A_{d}(z)$ are obtained as described by Rauhala and Välimäki. ${ }^{40}$ As suggested by Bank and Sujbert ${ }^{21}$ the decay times of the phantom partials are in the magnitude of the transversal vibrations. Hence, the loss filter parameters for $P(z)$ are copied from $S(z)$. The phantom partials start to become visible in the frequency domain only after the inharmonicity has shifted the transversal partials away from the phantom ones. In addition, high-frequency phantoms seem not to be very prominent. Therefore, $P(z)$ is filtered with a bandpass filter $D(z)$, made of fourth order high-and low-pass Chebyshev type I filters. In this work, $D(z)$ is designed to attenuate partials below the 10th partial and above the 20th.

To produce flageolet tones the parameters for $H_{\mathrm{LF}}(z)$ and $H_{R}(z)$ are designed with the following heuristic rules. The idea is to use the open string model with properly tuned ripple filter parameters. First, the $a$ parameter for $H_{\mathrm{LF}}(z)$ is calculated for a flageolet tone as described in Ref. 46. Then the target decay time $T_{60}$ for the fundamental frequency $f_{0}$ of the flageolet tone, obtained from the analysis, is used to determine the loop gain $g$ as

$$
g=e^{-\left(1 / f_{0} T_{60}\right)} .
$$

The ripple rate $R$ is obtained as

$$
R=\frac{1}{n},
$$

where $n$ is the harmonic index that functions as $f_{0}$ of the flageolet tone, i.e., $f_{n}=n f_{0}$. Then the ripple depth $r$ is calculated as

$$
r=e^{-\left(1 / f_{0} T_{60}^{\prime}\right)},
$$

where $T_{60}^{\prime}=T_{60} / 200$. This way the ripple filter strangles the decay times of the partials between the ringing ones to be approximately 200 times shorter than for $f_{0}$. To obtain the target decay time for the ringing partials $g$ is compensated by $1 /(r+1)$. This way the transfer function of the ripply loss filter is

$$
H_{\mathrm{LF}}(z) H_{R}(z)=\frac{g(1+a)}{r+1} \frac{r+z^{-R}}{1+a z^{-1}} .
$$

Stability is assured when the overall loop gain does not exceed unity. Due to scaling this is assured as long as $g<1$.

There are two advantages for using the ripple filter to produce flageolet tones. First, the open string excitation signal can be used, i.e., no separate excitation signal for flageolet tones is needed. Secondly, the tone of an open string can be changed to a flageolet tone simply by changing the ripple filter coefficients and not by changing $f_{0}$ of the string model.

\section{Results}

Next, synthesis results produced by the model discussed in Sec. IV A are shown, and the measured and synthesized signals and their significant characteristics are compared. 

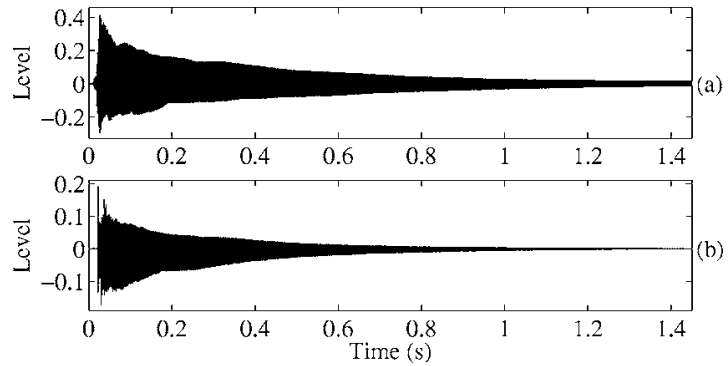

FIG. 14. Time responses of synthesized guqin tones played on string 6 mark 5 , when (a) the nail of the thumb terminates the string and (b) the fingertip of the forefinger terminates the string.

\section{Normal plucked tones}

Figure 14 depicts the time responses of synthesized signals for string 6 mark 5, for (a) nail and (b) fingertip terminations (compare with Fig. 5). The nail tone responses correspond fairly well, as do the fingertip tones, but they have a slight difference during the beginning of the decay. This can be explained by a difference in partial decay times, i.e., the higher frequencies of the real tone decay faster than the synthesized one. This can be improved with a higher loop filter order, ${ }^{48}$ but is left for future work. Next, the $T_{60}$ times for recorded and synthesized sounds, shown in Figs. 6 and 15, respectively, are looked at and compared. In both figures the decay times for string 1 is shown in (a) and for string 7 in (b), and the string termination style is indicated for nail $(+)$ and fingertip $(\bigcirc)$. For all synthesized tones the nail tones decay slower than for the fingertip terminated tones, as they should. The exception for both measured and synthesized is string 1 mark 13.1. The general trend is well preserved in the synthesis. However, synthesis results show a slight departure from the measured tones as a function of the mark, i.e., as the mark increases the difference between the synthesized and measured decay times becomes larger. This can be explained by the parameter smoothing discussed in Sec. IV B.

Figure 16 illustrates the splitting phenomenon produced with the proposed synthesis model (compare with Fig. 7). The splitting phenomenon becomes visible above the eighth partial. As expected, for the synthetic signals the locations of the modes are more systematic than for the measured cases. The inharmonicity of the synthesis follows target behavior
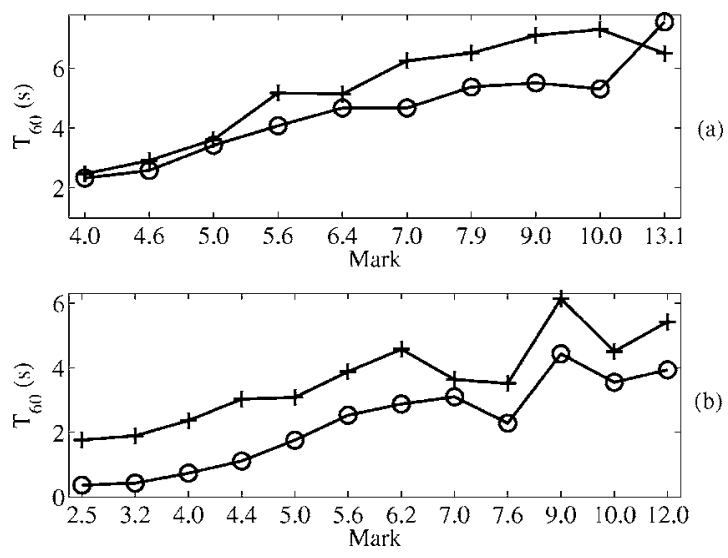

FIG. 15. $T_{60}$ times for all nail $(+)$ and fingertip $(\bigcirc)$ terminated synthetic tones for (a) string 1 and (b) string 7.
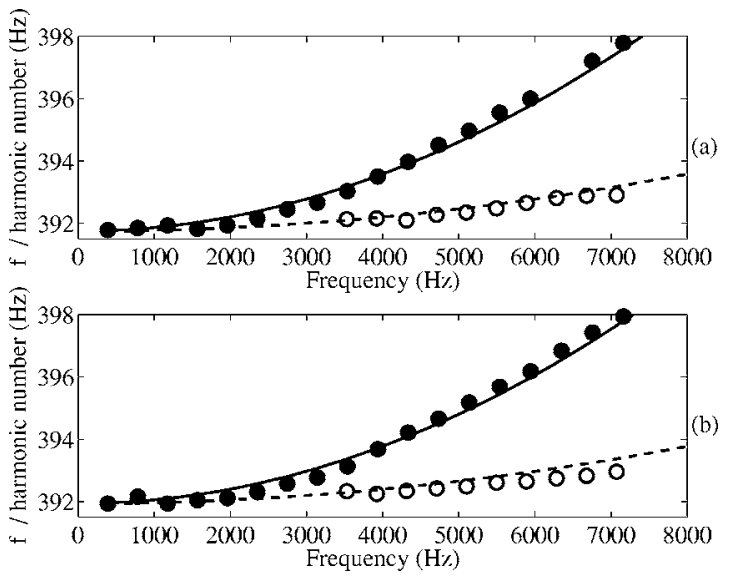

FIG. 16. Splitting of modes when synthesizing string 6 mark 5, for (a) nail and (b) fingertip termination tones. Target inharmonicity coefficient $B$ $=0.00009$ displayed with solid line and the white dots follow the $B / 4$ trend (dashed line). Measured $f_{0}$ values for tones in panels (a) and (b) are $391.74 \mathrm{~Hz}$ and $391.94 \mathrm{~Hz}$, respectively.

(solid and dashed lines) with a $0.5 \%$ error marginal up until the 20th partial. The perception of inharmonicity is a complex matter, ${ }^{34,49}$ and by interpreting the results obtained by Rocchesso and Scalcon ${ }^{49}$ the accuracy achieved with the used method ${ }^{40}$ should be adequate. The synthesis of phantom modes is further illustrated in Fig. 17 where magnitude responses of Figs. 17(a) and 17(b) measured and Figs. 17(c) and 17(d) synthesized string 6 mark 5 tones are shown. By comparing Figs. 17(b) and 17(d), one can see that the synthesis of the phantoms is successful. However, tuning of the inharmonicity filters causes some changes in the initial levels of higher harmonics. This is due to the strong variations in the spectrum of the excitation signal, which in proportion causes the changes in the levels of the harmonics. Panel (c) also shows the magnitude response of the bandpass filter $D(z)$ with a dashed line.

\section{Flageolet tones}

Figure 18 shows decay times regarding flageolet tone synthesis produced with the ripple filter for the fourth string and fifth harmonic. The target decay times are indicated with

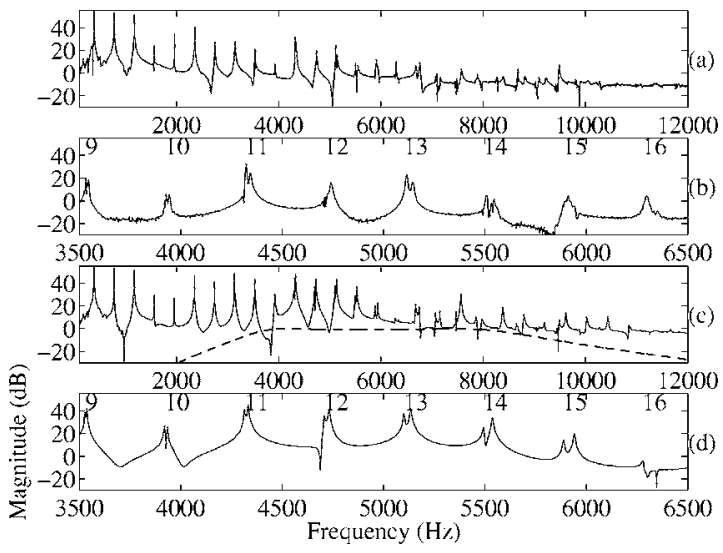

FIG. 17. Magnitude responses of (a-b) measured and (c-d) synthesized string 6 mark 5 tone. Panels (b) and (d) zoom into partials 9-16. The partial number is indicated by the number above. The response of bandpass filter $D(z)$ is also shown in pane (c) with a dashed line. 


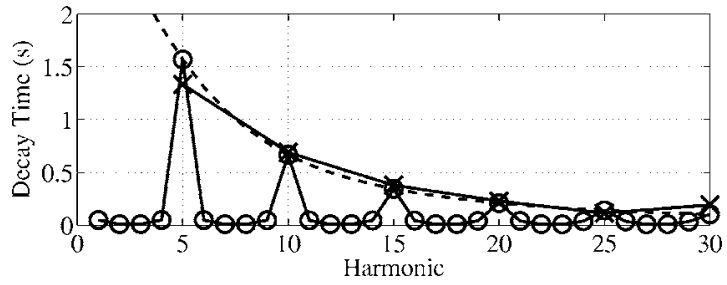

FIG. 18. Flageolet synthesis produced with the ripple filter. Target decay times are shown with $(\times)$ and synthesized with $(O)$. The dashed line indicates the decay time response of the loop filter without the ripple filter.

$\times$ marks and the synthesized ones with circles, the solid lines interpolate between the points. The dashed line illustrates the response of the loop filter without the ripple filter. The ripple filter is able to reduce the decay times of the partials between the ringing harmonics very well. As a consequence, a flageolet like tone can be produced with an open string synthesis model in the same way as for a real flageolet tone.

\section{Friction sounds}

The friction sound generator block (Fig. 12) is able to model the real finger-string friction sound relatively well. The spectrogram of a synthesized friction signal is shown in Fig. 19. For the synthesis control signal, a slide similar to that performed in Fig. 9(b) was applied. When comparing Figs. 9(b) and 19, many similarities can be seen. First, the overall shape of the noise is similar, a somewhat lowpass type, and its amplitude is highest when the slide velocity attains its peak. Also, both signals have a harmonic structure, the frequency of which also peaks with the slide velocity. The major difference between Figs. 9(b) and 19 is that the synthesized friction is obviously free of the measurement background noise, which hides some of the features in Fig. 9.

\section{SOFTWARE IMPLEMENTATION AND CONTROL OF THE GUQIN SYNTHESIZER}

The synthesis and control part of the guqin synthesizer is realized using a visual software synthesis package called PWGLSynth. ${ }^{30}$ The PWGLSynth is a part of a larger visual programming environment called PWGL. ${ }^{30}$ The control information is generated using the music notation package ENP. ${ }^{30}$ As this kind of work is experimental, and the synthesis model must be refined by interactive listening, a system is needed that is capable of making fast and efficient prototypes of the basic components of the system. The system used allows designing instrument models using a copying scheme for patches. Special synth-plug boxes are used in the graphical programming environment to automatically parameterize control entry points. Finally, a musical score is translated into a list of control events. The user can visually associate these events with the instrument definitions by using a mapping scheme. Since the approach used here is to control the synthesis model from the notation software package, a novel representation scheme of the ancient Chinese guqin Jian $\mathrm{Zi}$ $\mathrm{Pu}$ tabulature has been developed that is suitable for a modern computerized system. A coding system, developed by one of the authors (Henbing Li), based on Western latin characters is used to represent the left-hand and right-hand techniques found in the traditional guqin repertoire. The notation part can be enhanced with editable break-point functions, which allow the realization of the expressive pitch glides and vibrato gestures essential in guqin playing.

\section{CONCLUSIONS}

This paper discusses the acoustics and synthesis of the guqin, a traditional Chinese music instrument. The structural features and playing style of the guqin are discussed briefly. The proposed synthesis model is able to reproduce the important characteristics of the instrument, namely flageolet tones, friction sound due to sliding of the finger, different termination behavior, and the phantom partial series. The flageolet tones are produced with a ripply waveguide string model, so that the ripple filter parameters of an open string model are tuned to the desired harmonic. The friction sound is found to be of a harmonic nature and is synthesized with a model-based structure with a noise generator and three filters. The two termination techniques of pressed tones, fingernail and fingertip, cause a difference in decay character, so that tones terminated with the fingernail decay slower than those terminated with a fingertip. Analysis shows the existence of phantom partials. These are modeled with an auxiliary string model placed in parallel with the main string model. Agreement of the output of the synthesis model with measurements is not perfect, but prime features are well captured

and synthesized. Moreover, the model is computationally

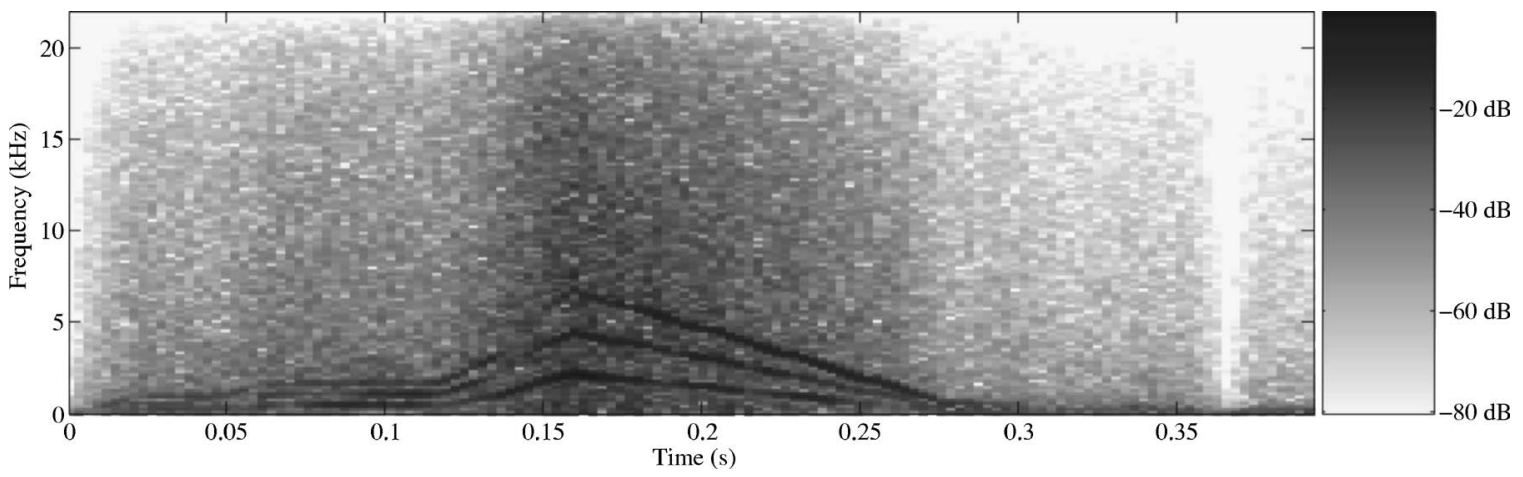

FIG. 19. Spectrogram of the synthesized friction noise when performing a similar slide as in Fig. 9(b). The figure shows that the synthesized friction sound is lowpass filtered noise with harmonic components. 
efficient enough to run in real-time. Measured and synthe sized guqin tones are available on the Internet at http:// www.acoustics.hut.fi/publications/papers/jasa-guqin/. In addition to capturing the prominent properties of the guqin, the model-based synthesis algorithm enables the stretching of reality and the realization of gliding harmonics and sliding of open strings, something that cannot be done in the real world.

A more detailed analysis of the behavior of the phantom partials and their parameterization for the waveguide model could improve the link of the model with the physical world. In addition, future research could include exact modeling of slides and vibrato. In the future, the proposed guqin model will be used for creating rule based guqin music from tabulatures, using rule-based control parameters.

\section{ACKNOWLEDGMENTS}

The authors are grateful to Jussi Pekonen, Jukka Rauhala, Hanna Järveläinen, Mika Kuuskankare, and Vesa Norilo for their help. Dr. Cumhur Erkut is thanked for his helpful comments. This work has been financially supported by COST287-ConGAS Action (Gesture Controlled Audio Systems), the Academy of Finland (Project No. 104934 and No. 105557), the Pythagoras Graduate School of Music and Sound Research, the Helsinki Graduate School of Electrical and Communications Engineering, and Tekniikan edistämissäätiö.

${ }^{1}$ The New Grove Dictionary of Music and Musicians, edited by S. Sadie (Oxford University Press, Oxford, 2001), pp. 630 and 677.

${ }^{2} \mathrm{R}$. V. Gulik, The Lore of the Chinese Lute (Charles E. Tuttle, Tokyo, 1969).

${ }^{3}$ M. Gimm, Qin, Die Musik in Geschichte und Gegenwart, edited by L. Fincher (Bärenreiter und Metzler, 1997), pp. 1916-1928.

${ }^{4}$ J. O. Smith, "Efficient synthesis of stringed musical instruments," in Proceedings of the International Computer Music Conference (ICMC'93) (Tokyo, Japan, 1993), pp. 64-71.

${ }^{5}$ M. Karjalainen, V. Välimäki, and Z. Jánozy, "Towards high-quality sound synthesis of guitar and string instruments," in Proceedings of the International Computer Music Conference (ICMC'93) (Tokyo, Japan, 1993), pp. $56-63$.

${ }^{6}$ J. O. Smith, "Physical modeling synthesis update," Comput. Music J. 20, 44-56 (1996), URL: http://www-ccrma.stanford.edu/ jos/pmupd/ pmupd.html. Last viewed on 14 September 2006.

${ }^{7}$ C. Erkut, M. Karjalainen, P. Huang, and V. Välimäki, "Acoustical analysis and model-based sound synthesis of the kantele," J. Acoust. Soc. Am. 112, 1681-1691 (2002).

${ }^{8}$ V. Välimäki, J. Pakarinen, C. Erkut, and M. Karjalainen, "Discrete-time modelling of musical instruments," Rep. Prog. Phys. 69, 1-78 (2006).

${ }^{9}$ A. W. Y. Su, W. C. Chang, and R. W. Wang, "An IIR synthesis method for plucked-string instruments with embedded portamento," Journal of the Audio Engineering Society 50, 351-362 (2002).

${ }^{10} \mathrm{H}$. A. Conklin, "Generation of partials due to nonlinear mixing in a stringed instrument," J. Acoust. Soc. Am. 105, 536-545 (1999).

${ }^{11}$ J. Pakarinen, "Physical modeling of flageolet tones in string instruments," in Proceedings of the 13th European Signal Processing Conference (EUSIPCO 2005) (Antalya, Turkey, 2005).

${ }^{12}$ V. Välimäki, H. Penttinen, J. Knif, M. Laurson, and C. Erkut, "Sound synthesis of the harpsichord using a computationally efficient physical model," EURASIP J. Applied Signal Processing 2004, 934-948 (2004).

${ }^{13}$ C. Canudas de Wit, H. Olsson, K. J. Åström, and P. Lischinsky, "A new model for control of systems with friction," IEEE Trans. Autom. Control 40, 419-425 (1995).

${ }^{14}$ P. Dupont, V. Hayward, and B. Armstrong, "Single state elasto-plastic models for friction compensation," IEEE Trans. Autom. Control 47, 787792 (2002).

${ }^{15}$ F. Avanzini, S. Serafin, and D. Rocchesso, "Interactive simulation of rigid body interaction with friction-induced sound generation," IEEE Trans. Speech Audio Process. 13, 1073-1081 (2005).

${ }^{16} \mathrm{~S}$. Serafin, "The sound of friction: Real-time models, playability and musical applications," Ph.D. thesis, Stanford University (2004), http:// www.imi.aau.dk/ sts/serafinthesis.pdf.

${ }^{17} \mathrm{P}$. Cook, "Physically informed sonic modeling (PhISM): Synthesis of percussive sounds," Comput. Music J. 21, 38-49 (1997).

${ }^{18}$ J. Pakarinen, M. Karjalainen, V. Välimäki, and S. Bilbao, "Energy behavior in time-varying fractional delay filters for physical modeling of musical instruments," in Proceedings of the IEEE International Conference on Acoustics, Speech, and Signal Processing (Philadelphia, PA, 2005), pp. $1-4$.

${ }^{19}$ I. Nakamura and D. Naganuma, "Characteristics of piano sound spectra," in Proceedings of the Stockholm Musical Acoustics Conference (Stockholm, Sweden, 1993), pp. 325-330.

${ }^{20}$ J. Woodhouse, "Plucked guitar transients: Comparison of measurements and synthesis," Acta. Acust. Acust. 90, 945-965 (2004).

${ }^{21}$ B. Bank and L. Sujbert, "Generation of longitudinal vibrations in piano strings: From physics to sound synthesis," J. Acoust. Soc. Am. 117, $2268-$ 2278 (2005).

${ }^{22}$ B. Bank and L. Sujbert, "Modeling the longitudinal vibration of piano strings," in Proceedings of the Stockholm Musical Acoustics Conference (Stockholm, Sweden, 2003), pp. 143-146.

${ }^{23} \mathrm{~J}$. Bensa and L. Daudet, "Efficient modeling of phantom partials in piano tones," in Proceedings of the International Symposium in Musical Acoustics (Nara, Japan, 2004), pp. 207-210.

${ }^{24} \mathrm{G}$. Carrier, "On the nonlinear vibration problem of the elastic string," $\mathrm{Q}$. Appl. Math. 3, 157-165 (1945).

${ }^{25} \mathrm{C}$. Valette, The Mechanics of Vibrating Strings (Springer, New York, 1995), pp. 116-183.

${ }^{26}$ T. Tolonen, V. Välimäki, and M. Karjalainen, "Modeling of tension modulation nonlinearity in plucked strings," IEEE Trans. Speech Audio Process. 8, 300-310 (2000).

${ }^{27}$ B. Glasberg and B. Moore, "Deviation of auditory filter shapes from notched noise data," Hear. Res. 47, 103-138 (1990).

${ }^{28} \mathrm{H}$. Järveläinen, "Perception of attributes in real and synthetic string instrument sounds," Ph.D. thesis, Helsinki University of Technology, Espoo, Finland (2003), http://lib.tkk.fi/Diss/2003/isbn9512263149/. Last viewed on 14 September 2006.

${ }^{29}$ A. Cheveigné and H. Kawahara, "YIN, a fundamental frequency estimator for speech and music," J. Acoust. Soc. Am. 111, 1917-1930 (2002).

${ }^{30}$ M. Laurson, V. Norilo, and M. Kuuskankare, "PWGLSynth, a visual synthesis language for virtual instrument design and control," Comput. Music J. 29, 29-41 (2005).

${ }^{31}$ H. Järveläinen, V. Välimäki, and M. Karjalainen, "Audibility of the timbral effects of inharmonicity in stringed instrument tones," Acoust. Res. Lett. Online (ARLO) 2, 79-84 (2001).

${ }^{32}$ H. Fletcher, E. D. Blackham, and R. Stratton, "Quality of piano tones," J. Acoust. Soc. Am. 34, 749-761 (1962).

${ }^{33}$ N. H. Fletcher and T. D. Rossing, The Physics of Musical Instruments, 2nd ed. (Springer-Verlag, New York, 1998), p. 620.

${ }^{34} \mathrm{M}$. Karjalainen and H. Järveläinen, "Is inharmonicity perceivable in the acoustic guitar?," in Proceedings of Forum Acusticum 2005 (Budapest, Hungary, 2005), pp. 435-440.

${ }^{35}$ B. C. J. Moore, R. W. Peters, and B. R. Glasberg, "Thresholds for the detection of inharmonicity in complex tones," J. Acoust. Soc. Am. 77, 1861-1867 (1985).

${ }^{36}$ D. Jaffe and J. O. Smith, "Extensions of the Karplus-Strong plucked string algorithm," Comput. Music J. 7, 56-69 (1983), reprinted in The Music Machine, edited by C. Roads (MIT Press, Cambridge, 1983), pp. 481-494.

${ }^{37}$ S. A. Van Duyne and J. O. Smith, "A simplified approach to modeling dispersion caused by stiffness in strings and plates," in Proceedings of the International Computer Music Conference (ICMC'94) (Aarhus, Denmark, 1994), pp. 407-410.

${ }^{38}$ D. Rocchesso and F. Scalcon, "Accurate dispersion modeling for piano strings," in Proceedings of the Nordic Acoustical Meeting (Helsinki, Finland, 1996), pp. 407-414.

${ }^{39}$ B. Bank, F. Avanzini, G. Borin, G. De Poli, F. Fontana, and D. Rocchesso, "Physically informed signal-processing methods for piano sound synthesis: A research overview," EURASIP J. Applied Signal Processing 2003, 941-952 (2003), special issue on Digital Audio for Multimedia Communications.

${ }^{40} \mathrm{~J}$. Rauhala and V. Välimäki, "Tunable dispersion filter design for piano synthesis,” IEEE Signal Process. Lett. 13, 253-256 (2006). 
${ }^{41}$ M. Karjalainen, V. Välimäki, and T. Tolonen, "Plucked-string models: From the Karplus-Strong algorithm to digital waveguides and beyond," Comput. Music J. 22, 17-32 (1998).

${ }^{42}$ S. Bilbao, "Time-varying generalizations of all-pass filters," IEEE Signal Process. Lett. 12, 376-379 (2005).

${ }^{43} \mathrm{~J}$. A. Moorer, "The use of linear prediction of speech in computer music applications," Journal of the Audio Engineering Society 27, 134-140 (1985).

${ }^{44}$ U. Zölzer, Digital Audio Signal Processing (Wiley, Chichester, 1997), p. 279.

${ }^{45}$ D. Rocchesso and J. O. Smith, "Circulant and elliptic feedback delay networks for artificial reverberation," IEEE Trans. Speech Audio Process. 5, 51-63 (1997).
${ }^{46} \mathrm{~V}$. Välimäki and T. Tolonen, "Development and calibration of a guitar synthesizer," J. Audio Eng. Soc. 46, 766-778 (1998).

${ }^{47}$ V. Välimäki, J. Huopaniemi, M. Karjalainen, and Z. Jánozy, "Physical modeling of plucked string instruments with application to real-time sound synthesis," Journal of the Audio Engineering Society 44, 331-353 (1996). ${ }^{48}$ H.-M. Lehtonen, J. Rauhala, and V. Välimäki, "Sparse multistage loss filter design for waveguide piano synthesis," in Proceedings of the IEEE Workshop on Applications of Signal Processing to Audio and Acoustics (New Paltz, New York, 2005), pp. 331-334.

${ }^{49}$ D. Rocchesso and F. Scalcon, "Bandwidth of perceived inharmonicity for physical modeling of dispersive strings," IEEE Trans. Speech Audio Process. 7, 597-601 (1999), URL: http://profs.sci.univr.it/ rocchess/. Last viewed on 14 September 2006. 